Instituto Internacional de Investigación y Desarrollo Tecnológico Educativo INDTEC, C.A.

DOI: https://doi.org/10.29394/scientific.issn.2542-2987.2017.2.4.12.204-222

OAI-PMH: http://www.indteca.com/ojs/index.php/Revista Scientific/oai

\title{
Producción de Cultivo Hidropónico Lechuga (Lactuca Sativa L.) para la Promoción de la Autogestión en la Escuela Básica Bolivariana "Los Naranjos"
}

\author{
Autor: Luis Alfredo Díaz \\ Escuela Básica Bolivariana "Los Naranjos" \\ luis.a.godoy@hotmail.com \\ Barinas, Venezuela
}

Resumen

La presente investigación tiene como propósito la promoción del Cultivo Hidropónico Lechuga (Lactuca Sativa L.) como alternativa de autogestión en la Escuela Básica Bolivariana "Los Naranjos", parroquia Calderas, municipio Bolívar del estado Barinas. La naturaleza del estudio está enmarcada bajo la modalidad del paradigma cualitativo, adoptando el método de Investigación Acción Participativa. Por otro lado, la investigación se realizará en (4) cuatro fases: Fase I. Diagnóstica, Fase II. Elaboración del plan de acción, Fase III. Ejecución del plan de acción, Fase IV. Reflexión, interpretación e integración de resultados. Para el análisis de la información se utilizó la triangulación de información con el fin de realizar el cruce de información de los informantes claves. Por todo lo expuesto, este estudio constituye un aporte significativos al proceso de enseñanza y aprendizaje ya que es competencia de la familia y de los docentes como promotores y agentes de difusión de conocimientos, aceptar la responsabilidad en este proceso de colocar en contacto a los niños y niñas con nuevas técnicas de cultivo, en este caso con la técnica hidropónica, desde sus primeros años, para que esta sea una experiencia placentera, grata y alentadora que lo acompañe por el resto de su vida. flotante.

Palabras clave: cultivo hidropónico; autogestión; sistema de raíz 


\title{
Lettuce Production Hydroponics (Lactuca Sativa L.) for the Promotion of Self-Management in Basic School Bolivariana "Los Naranjos"
}

\begin{abstract}
The present research aims at the promotion of the Hydroponic Lettuce Cultivation (Lactuca Sativa L.) as a self - management alternative in the Bolivarian Basic School "Los Naranjos", Calderas parish, Bolívar municipality of Barinas state. The nature of the study is framed under the modality of the qualitative paradigm, adopting the method of Participatory Action Research. On the other hand, the research will be conducted in (4) four phases: Phase I. Diagnosis, Phase II. Elaboration of the action plan, Phase III. Implementation of the action plan, Phase IV. Reflection, interpretation and integration of results. Information triangulation was used to analyze the information in order to crosscheck information from key informants. For all of the above, this study constitutes a significant contribution to the teaching and learning process as it is the responsibility of the family and teachers as promoters and agents of dissemination of knowledge, accept the responsibility in this process of placing children in contact And girls with new techniques of cultivation, in this case with the hydroponic technique, since its early years, so that this is a pleasurable, pleasant and encouraging experience that accompanies him for the rest of his life.
\end{abstract}

Keywords: hydroponics; self-management; floating root system.

Date Received: 05-10-2016

Date Acceptance: 07-11-2016 


\section{A Modo de Introducción}

La agricultura en Venezuela es el arte y la ciencia para cultivar la tierra, obteniendo de ella la máxima producción con la mayor economía posible. Su importancia radica en que ella, constituye la base de la presencia y permanencia del hombre sobre la tierra, por lo que la misma aporta los alimentos que necesita el ser humano para subsistir.

Los huertos escolares son una poderosa herramienta para mejorar la calidad de la nutrición y la formación de los niños, niñas y adolescentes y sus familias en las zonas rurales y urbanas de los países en desarrollo. Por eso es importante, citar a Boff (2013), quien refiere que la sostenibilidad, plantea:

Como actualmente se ha llegado a un nivel tan elevado de agresión que la humanidad le ha declarado una guerra total al planeta tierra, en el suelo, en el subsuelo, en el aire, en el mar, en las montañas, en los bosques, en los reinos animales y vegetal en cualquier lugar donde se pueda arrancarle algo para nuestro propio beneficio, sin ningún sentido, de retribución ni disposición alguna de concederle reposo y tiempo para regenerarse (pág. 26).

En vista de la situación descripta anteriormente, el futuro mundial de Latinoamérica y la humanidad depende de la respuesta que el Estado promueva para todos los países tanto en materia de legislación y de nuevas alternativas de producción, es decir, apoyar en reconocer la axiología con el objeto de fomentar las aptitudes y actitudes necesarias para comprender las interrelaciones entre el hombre, su cultura y su medio físico. Por otro lado, Barragán (2010) plantea:

Que el hombre desde sus orígenes ha alterado el equilibrio natural, afectando en forma negativa los componentes del ambiente (biótico y abiótico), en donde se desenvuelve (pág. 34).

Por consiguiente, en las últimas décadas, se ha visto con mucha preocupación como se ha degradado progresivamente el ambiente y con ello 
destruyendo los recursos naturales los cuales son la base donde se produce la satisfacción de las necesidades para las generaciones futuras. No obstante, hay que hacer notar que las alteraciones ambientales, manifestadas antes del advenimiento de la revolución industrial, el crecimiento exponencial de la población, y el acelerado proceso de urbanización que desarrolla el género humano, la naturaleza tenía la capacidad de autorregularse, sin embargo, hoy día podemos expresar la posibilidad de provocar una ruptura ambiental sin precedente.

De lo anteriormente se deduce, que los huertos escolares en la actualidad son una excelente estrategia para convertir los centros educativos en lugares que posibiliten a los estudiantes, múltiples experiencias acerca de su entorno así como colocar en práctica hábitos y valores de responsabilidad, siendo una herramienta que no solo genere entusiasmo entre los docentes y estudiantes sino que además permita complementar la oferta educativa brindando propuestas y actividades que sean de gran interés para ampliar el trabajo y profundizar los contenidos. Al respecto, Trujillo (2000) expresa:

Un huerto puede crearse en cualquier espacio, bien sea en una terraza, parcela de tierra, un balcón, macetero de grandes dimensiones y profundidad. Se deben construir en lugares altos para poder vigilarlos con facilidad y prestarles los cuidados necesarios, la orientación que se le debe dar es siguiendo la orientación del curso del sol, es decir, de este a oeste evitando de esta manera la acción de los rayos solares durante todo el día (pág. 20).

Entonces, es una reciprocidad entre el docente que posee los saberes y la experiencia para orientar el aprendizaje y un grupo estudiantes quienes a través de sus estudios incorporan progresivamente esos conocimientos a su ámbito de competencia y en ese tránsito van conformando su aprendizaje y propician el ambiente y los recursos para construir, desafiar, revisar y reestructurar los conocimientos nuevos. 
Ante la situación planteada, la hidroponía, es una técnica relativamente nueva en nuestro medio para producir cultivos saludables. Esta técnica permite cosechas en períodos más cortos que la siembra tradicional, mejor sabor y calidad del producto, mayor homogeneidad y producción.

En este sentido, la presente investigación está enmarcada en una investigación acción participativa (IAP), bajo un abordaje metodológico de tipo cualitativo que permitió estructurar algunos elementos desde la interpretación, sentimientos, opiniones y significados de los entrevistados desde sus experiencias para aportar algunos constructos teóricos-prácticos para contribuir a desarrollar la producción de cultivo hidropónico lechuga (Lactuca Sativa L.) para la promoción de la autogestión en la Escuela Básica Bolivariana "Los Naranjos".

Es evidente, que a partir del año escolar 2003-2004, se comienza sentir y observarse la preocupación por la problemática a través de diálogos y conversaciones entre el colectivo docente; quienes han señalado las debilidades de desarrollar métodos alternativos para aprovechar los espacios para desarrollar los cultivos hidropónicos que irían al Sistema de Alimentación Escolar Bolivariana; cuya observación y preocupación se hace más evidente en las ochos (8) instituciones adscrita al Núcleo Escolar Rural № 187. De igual manera, los docentes de desarrollo endógeno emplean los mismos métodos y técnicas de enseñanzas tradicionales muchas veces sin comprensión; que en vez de promover el acercamiento hacia la agricultura alternativa, lo que generan es rechazo de los niños, niñas y adolescentes hacia esta actividad socio productiva.

Es importante señalar que en la Escuela Básica Bolivariana "Los Naranjos", ubicada en el municipio Bolívar, parroquia Calderas, estado Barinas, la elaboración y ejecución de un huerto escolar pudieran permitir estudiar e integrar sistemáticamente ciclos y procesos dinámicos de los fenómenos naturales, valores, realidades sociales, integración y relaciones 
que componen el sistema. Además, de crear una concientización sobre el ambiente y se constituya en un espacio donde los estudiantes los padres y/o representantes se acerquen a trabajar de manera espontánea y autónoma con la debida guía y orientación de los docentes

Por lo tanto, la autogestión comunitaria es un enfoque integral de la vida en sociedad, que no solo se refiere a organizar de manera democrática o participativa el lugar de trabajo o la empresa productora de bienes o servicios que se extiende a todas las dimensiones de la vida económica y social en comunidad. La base de la autogestión está en el concepto mismo de la persona (o del ser humano) y sus derechos fundamentales, como el derecho a la autodeterminación y a la participación, en las esferas personales, familiares y comunitarias.

Como ya se ha aclarado, esta investigación se considera relevante porque orienta y promueve a los escolares a la realización de un cultivo hidropónico a través de estrategias y actividades en el ámbito escolar, familiar y comunitario con el propósito de mejorar la situación de las pocas acciones de autogestión por parte de la comunidad educativa en la Escuela Básica Bolivariana "Los Naranjos", ubicada en el municipio Bolívar, parroquia Calderas, estado Barinas.

De esta manera, se minimizan los problemas de la autogestión que cada vez se presentan en mayor cantidad a nivel de la comunidad educativa y haciendo así más fácil y efectivo el proceso para el aprendizaje, el desarrollo personal, social y profesional de los niños, niñas y adolescentes de esta población en lo que respeta a la producción de otros rubros para las instituciones.

En vista de los planteamientos anteriores, surgen las siguientes interrogantes que orientan la investigación:

1. ¿Cuáles son los conocimientos sobre la producción de cultivos hidropónicos que poseen los niños, niñas y adolescentes de la Escuela 
Básica Bolivariana "Los Naranjos"?

2. ¿Cómo sería el diseño de la estrategia para la producción de cultivos hidropónicos en la Escuela Básica Bolivariana "Los Naranjos"?

3. ¿Cómo se desarrollará la estrategia para la producción de cultivos hidropónicos en la Escuela Básica Bolivariana "Los Naranjos"?

4. ¿Cómo evaluar la producción de los cultivos hidropónicos y la autogestión en la Escuela Básica Bolivariana "Los Naranjos"?

\subsection{Propósito General de la Investigación}

Promover la producción del Cultivo Hidropónico (Lactuca Sativa L.) como alternativa de autogestión en la Escuela Básica Bolivariana "Los Naranjos", parroquia Calderas, municipio Bolívar del estado Barinas.

\section{Teorías y Conceptos}

Al respecto, Bavaresco (2006), expresa que el marco de sustentación, brinda a la investigación un sistema coordinado y coherente de conceptos y proposiciones que permiten abordar el problema dentro de un ámbito dónde éste cobre sentido (pág. 213). A continuación, se presenta un estudio internacional como referencia en la presente investigación.

Por su parte, Avalos (2014), en su Tesis de Maestría en Formulación y Evaluación de Proyectos Titulado: Los Invernaderos como Alternativa para Optimizar el Uso del Suelo y Elevar los Rendimientos de Cosechas: Caso del Cultivo de Tomate. La investigación tuvo como objetivo general, aportar prácticas alternativas de producción a través del sistema hidropónico en condiciones de invernadero, como una opción novedosa para el desarrollo de la agricultura en las áreas peri-urbanas y rurales de Guatemala.

Según el autor, se utilizó el enfoque descriptivo/exploratorio fue posible demostrar que, utilizando materiales de bajo costo en la construcción del invernadero y el sistema hidropónico, se logró una disminución significativa en 
la inversión inicial del proyecto, contando con la ventaja de que los materiales utilizados en este estudio se caracterizan por encontrarse disponibles permanentemente en el mercado.

Ante la situación planteada, los resultados obtenidos del trabajo de investigación son los que se pueden apreciar a continuación: El desarrollo del estudio confirma que al utilizar materiales alternativos para la construcción de un invernadero, se obtuvieron rendimientos de cosecha superiores comparados con la utilización de prácticas agrícolas tradicionales, ya que la aplicación de solución nutritiva que circula en los canales de riego permite a las plantas un fácil acceso a los nutrientes disueltos en dicha solución.

La relación de la investigación con el presente estudio se fundamenta en que se maneja dos categorías importantes para el desarrollo del proyecto de investigación sistema hidropónico en condiciones de invernadero y el sistema raíz flotante, lo cual fortaleció el estudio, ya que se utilizó esta técnica como medio de producción para que los estudiantes y la comunidad sienta la curiosidad y el interés de sembrar lechugas con este método novedoso en el caserío "Los Naranjos", permitiendo así obtener más cosechas que los cultivos tradicionales.

\subsection{Perspectiva Teórica}

Según Méndez (2001), el referente teórico es la descripción de los elementos teóricos planteados por uno o por diferentes autores que permiten al investigador fundamentar los procesos de conocimientos con dos aspectos diferentes: Así mismo, permite ubicar el tema objetivo de investigación dentro de las teorías que serán directamente utilizados en el desarrollo de la investigación, incluyendo las relaciones más significativas que dan entre esos elementos teóricos (pág. 246).

Por otro lado, es una descripción detallada de cada uno de los elementos de la teoría que serán directamente utilizados en el desarrollo de la 
investigación, incluyendo las relaciones más significativas que se den entre esos elementos teóricos. En tal sentido, en el presente estudio se efectuó la revisión de algunas teorías relacionadas con los cultivos hidropónicos y la autogestión.

\subsection{Cultivos Hidropónicos}

Para Marulanda (2007), la palabra hidroponía se deriva del griego Hydro (agua) y Ponos (labor, trabajo) lo cual significa literalmente trabajo en agua. Esta definición se usa en la actualidad para describir todas las formas de cultivos sin suelo. Esta técnica hidropónica se ha puesto en práctica bajo diferentes modalidades: raíz en sustrato solido (mezcla de materiales como arena, carbón, granza de arroz, entre otros), raíz en agua o raíz desnuda, por esta razón la palabra hidroponía se entiende hoy como el "cultivo sin tierra" (pág. 15).

Entonces se puede aseverar, que la hidroponía está comenzando a consolidarse en América Latina como una alternativa en la lucha contra la pobreza. En muchos países constituye parte de la base de programas nacionales; en otros se encuentra todavía en proceso de desarrollo. Representa, sin lugar a dudas, una opción en la mejora del ingreso y de la calidad de vida, que maximiza los componentes de la información, a la vez que reduce a un mínimo la inversión, ofreciendo una alternativa sostenible de desarrollo.

\subsection{Importancia de los Cultivos Hidropónicos}

En la actualidad, la hidroponía como tecnología aplicada, puede ser considerada como una alternativa eficaz, para ser integrado a la producción agrícola en zonas rurales y urbanas, reconociendo que el uso de la hidroponía simplificada produce frutos y hortalizas de buena calidad, sin contaminación, frescas, en mejores condiciones de nutrición en contraposición a las que 
pueden accederse en los comercios. Desde esta perspectiva, Marulanda (2003), manifiesta:

La hidroponía juega un papel de suma importancia siendo considerada como un sistema de producción agrícola que tiene gran valor dentro del contexto ecológico, económico y social. Dicha importancia se basa en la gran flexibilidad del sistema, es decir, por la posibilidad de aplicarlo con éxito, bajo muy distintas condiciones y para diversos usos. Con estos criterios se puede decir que se satisfacen las necesidades esenciales de la generación presente sin comprometer la capacidad de satisfacer las necesidades esenciales de las generaciones futuras (pág. 48).

Siguiendo el mismo orden de idea, la agricultura en la zona rural debe considerarse como fundamental la preservación de los recursos naturales, con el objeto de generar modelos de explotación racional y eficiente. Teniendo como fin último lograr la equidad y la justicia en la distribución de la riqueza, el desarrollo económico, la producción y el consumo eficaz de los recursos naturales.

\subsection{Sistema de Raíz Flotante}

Para Rodríguez (2004), los sistemas de cultivos de raíz flotante han sido encontrados eficientes para el cultivo de albahaca, cebollín, apio y varios tipos de lechuga, con excelentes resultados, en ahorro de tiempo y altas producciones. Por lo que este autor manifiesta:

Este método utiliza agua y sales nutritivas, es muy importante recordar que por lo menos dos veces al día, se debe levantar con cuidado y agitar el agua con la mano hasta formar burbujas de aire, ya que las plantas al igual que nosotros, necesitan de aire para sobrevivir. El agua dentro de la caja debe cambiarse una vez al mes, es decir, se saca el agua de la caja y se vuelve a hacer el procedimiento indicado para alimentar las plantas con el método de raíz flotante (pág. 21).

En síntesis, la producción de lechuga bajo este sistema consiste en que 
las raíces están sumergidas en solución nutritiva, las plantas se encuentran en planchas de poliestireno expandido que flotan sobre el agua con la solución nutritiva en donde la plancha actúa como soporte mecánico y cada una flota sosteniendo un determinado número de plantas de lechuga

\subsection{Autogestión}

En este contexto, para Lama (2007), expresa que el termino autogestión se refiere al uso de cualquier método, habilidad y estrategia a través de las cuales los individuos puedan dirigir eficazmente sus propias actividades hacia el logro de sus objetivos. Incluye el establecimiento de metas, planificaciones, programas, seguimiento de tareas (pág. 14).

Es importante acotar que, si el Caserío "Los Naranjos" aspira a la autogestión y el autodesarrollo comunitario como vías de sostenibilidad de los proyectos, se precisa sensibilizar e involucrar a instituciones de gobierno, autoridades locales y a la propia comunidad en las acciones que se desprenden del estudio.

Entonces, para el desarrollo de la autogestión es importante considerar la participación de los entes involucrado en el proyecto de investigación, con el propósito que la comunidad educativa participen en la construcción del estudio con compromiso social, con honestidad y con la intensión de informar, comunicar o notificar, es decir, participar significa la libertad que tiene un individuo para dedicarse a cualquier actividad productiva, social o comunitaria con la intención de buscar las posibles soluciones en pro del caserío "Los Naranjos".

\section{Perspectiva Metodológica}

Al abordar el trabajo de investigación, es necesario identificar la metodología adecuada para lograr los propósitos propuestos. Es competencia del investigador definir la naturaleza del estudio, su tipo o modalidad, el diseño 
de la investigación, la selección del método, la recolección, análisis de la información, las técnicas e instrumentos de recolección de datos y la interpretación de respuestas brindadas en cuestionarios o entrevistas realizadas.

Por otro lado, una vez finalizada la perspectiva metodológica y la revisión bibliográfica, el siguiente paso es mantener la credibilidad y confirmabilidad, con el fin de dar respuestas en forma ordenada y sistemática a las interrogantes planteadas, con el propósito de tener una visión clara de lo que se hizo, por qué y cómo se hizo. Además, deben mencionarse las razones por las cuales se seleccionó dicha metodología, su adecuación al problema en estudio, para posteriores trabajos de investigación.

\subsection{Naturaleza de la Investigación}

El estudio se sustenta en el paradigma cualitativo; históricamente, la investigación cualitativa se origina del contexto de la antropología cultural centrada inicialmente en el estudio de pequeños grupos humanos. (Rojas, 2010) establece que:

Un investigador cualitativo debe ver a la sociedad desde una perspectiva integral, con un enfoque holístico, prestando atención, además, a los individuos no como objetos de estudio solamente, sino como un todo en sí mismos, debido a que este enfoque se apega de buena forma a la subjetividad del ser, rompiendo un poco con la visión rígida del positivismo y abriéndose paso entre las ciencias humanistas, pero sin dejar de lado la objetividad al momento del análisis de los datos hallados (pág. 11).

Entonces, el paradigma consiste en descripciones detalladas de situaciones, eventos, personas, interacciones y comportamientos que son observables. Incorpora lo que los participantes dicen, sus experiencias, actitudes, creencias, pensamientos y reflexiones tal como son expresadas por ellos mismos y no como uno los describe. Es importante precisar que el 
enfoque cualitativo es inductivo, es decir, se necesita conocer el terreno que estamos pisando.

\subsection{Tipo o Modalidad de Investigación}

El problema que motiva este trabajo de investigación se ajusta al esquema de Investigación Acción Participativa (IAP), al respecto Hurtado (1998), establece:

La investigación social es una modalidad de investigación interactiva, que se caracteriza por que la acción surge como una necesidad de un grupo o comunidad y es realizada o ejecutada por los involucrados en la situación a modificar, de tal manera que el investigador actúa como facilitador del proceso. Por otro lado, según esta misma autora, el propósito de la investigación acción "consiste en modificar el sistema estudiado, generando y aplicando sobre él una intervención especialmente diseñada" (pág. 92).

De lo anteriormente plantado, el investigador debe confrontar la realidad, la teoría formulada y de alguna manera obtener y registrar la información proveniente de esa realidad o fenómenos de estudio, surgiendo entonces la recolección de información o datos, así mismo, se reflexionó sobre los recursos financieros disponibles, el equipo humano que realizó la investigación y la cooperación que se espera lograr de los habitantes de la comunidad "Los Naranjos".

Por otra parte, para la confiabilidad de la información se realizó una triangulación desde la óptica de los informantes claves conformada por tres (3) representantes del caserío "Los Naranjos". Estos informantes claves o actores sociales, son las personas que hacen vida en la comunidad afectada y poseen información relevante para el estudio de la problemática a tratar en la Escuela Básica Bolivariana "Los Naranjos", que son las pocas acciones por parte de los habitantes de la comunidad por buscar alternativas para realizar cultivos con otras técnicas novedosas. 
Es importante acotar que las técnicas utilizadas en el trabajo de campo fueron la observación participante, la entrevista a profundidad con el propósito de explorar, describir ambiente e ingresar en profundidad, en situaciones sociales y mantener un rol activo, pendiente de los detalles, actitudes, creencias, situaciones, sucesos e interaccione, donde los investigadores reciben datos directos, sobre todo descriptivos, participando en la vida cotidiana del grupo, de una organización y de las personas que van a estudiar.

Entre los instrumentos de investigación se utilizaron la entrevista a expertos, notas de campo, registro fotográfico permitiendo la recolección de los distintos procedimientos, actividades que permiten al investigador obtener la información necesaria y objetiva para dar respuestas a las preguntas de investigación y se genere un acercamiento entre el investigador y los informantes,

Los recursos empleados en la investigación fueron, materiales, tales como, equipos de computación, material de oficina, cámara fotográfica, teléfonos celulares, cámara filmadora, grabadoras, transporte, fotocopias y de logística humanos: personal directivo, docentes, padres y representantes, niños, niñas y adolescentes, entrevistados, especialista en el área, personal de apoyo e investigador Finalmente, el procedimiento de recogidas de información es para analizar o dar sentido a los datos cualitativos para reducir notas de campos, descripciones, explicaciones y justificaciones como evidencia del trabajo realizado.

\section{Reflexiones Finales y Recomendaciones}

\subsection{Reflexiones Finales}

Para desarrollar los cultivos hidropónicos como alternativa de autogestión, es importante tener presente la integración de los niños, niñas, adolescentes y comunidad en general a las diversas actividades a desarrolladas tomando en cuenta el contexto familiar y social de la comunidad 
"Los Naranjos" permitiéndole enriquecer su praxis agrícola en materia educativa.

Esta investigación se elaboró con la intención de motivar a las familias y escuela de las zonas rurales, para que en esas áreas del jardín o patio, poco o nada utilizada; inicien un pequeño proyecto hidropónico. El esfuerzo es mínimo y la satisfacción mucha, sobre todo para aquellos y aquellas que gustan de las hortalizas. La facilidad del manejo, el poco tiempo requerido y la calidad de la cosecha, no solo benefician a la familia en cuanto al consumo de alimentos frescos y sanos, sino que es una actividad que puede ser compartida por todo el grupo familiar, pues permite integrar al adulto mayor y a los jóvenes, a nueva experiencia, disciplina y el hábito de trabajo en grupo. Una vez finalizado el trabajo, se llegan a las siguientes reflexiones finales:

1. En cuanto a la fase diagnóstica, el personal docente debe aplicar metodologías innovadoras y creativas, para superar el desinterés y el rechazo que sienten los alumnos hacia la producción de diversas hortalizas, ya que se evidencio que los educadores, padres y/o representantes, no buscan los métodos y técnicas para salir de la rutina de estos cultivos tradicionales.

2. Como resultado de la investigación, sobre diseñar estrategias para la producción del cultivo hidropónico, se expone a continuación de manera general las diversas opiniones y sentimientos del personal docente, padres, representantes y alumnos, donde se reconoce que la producción de hortaliza tales como: (lechuga, tomate, ají dulce otros), aporta beneficio para la salud, al ámbito académico y social, debido a que pueden ser manejadas por cualquier miembro del grupo familiar.

3. Es importante, reflexionar que a través de la técnica hidropónica logramos consumir alimentos: Libres de contaminantes químicos y de baterías. Así mismo, manifestaron que a través del método de raíz flotante se puede lograr el autoabastecimiento familiar con diversas 
hortalizas de calidad y a bajo costo. Es importante resaltar, que a través de las estrategias y del método de raíz flotante las hortalizas cultivas tanto en la escuela como en el hogar de cada representante son una fuente valiosa de alimento y hasta una contribución nutricional importante en la dieta balanceadas de cada ser humano. Dichos cultivos hidropónicos a través de la práctica en la escuela pueden ser trabajados por el grupo familiar y con la participación de mujeres, niños, niñas y adolescentes con necesidades educativas especiales.

\subsection{Recomendaciones}

De acuerdo a las conclusiones expuestas anteriormente y con el propósito de optimizar el funcionamiento de la Producción de los cultivos hidropónico (Lactuca Sativa L) para la promoción de la autogestión en la Escuela Básica Bolivariana "Los Naranjos" se proponen las siguientes recomendaciones a la comunidad en general: Es pertinente que los alumnos y alumnas de la Escuela Básica Bolivariana "Los Naranjos" se les enseñe que los cultivos hidropónicos es una técnica innovadores que con el pasar del tiempo será el cultivo del futuro para promover la sustentabilidad y preservar la biodiversidad en la instituciones educativa como también en las comunidades adyacentes.

1. Los alumnos pueden, gracias a la producción de los cultivos hidropónicos, desarrollar aprendizajes conceptuales, actitudinales y procedimentales en el área de las ciencias naturales, así como desarrollar valores: como la solidaridad, cooperación y respeto entre compañeros y despertar el interés hacia la protección del ambiente, y al mismo tiempo escapar de la rutina de las actividades centradas en el aula; de forma que deberían solicitar a sus docentes y al personal directivo de las instituciones educativas, la inclusión de actividades de esta índole. 
2. Incentivar a los padres y madres que participen activamente en el diseño de las acciones para poner en marcha la escuela, facilitando la ejecución de la propuesta a través del nombramiento de un responsable por la directora de la institución para seguir despertando el interés por la producción de los cultivos hidropónicos.

3. El personal directivo, docente, e investigador al diseñar el huerto hidropónico debe garantizar la conservación y preservación de los espacios ejerciendo la defensa a través del marco legal ambiental tanto nacional como internacional en pro de nuestra biodiversidad.

4. Mejorar los planes y programas educativos y ecológicos donde los actores educativos se sensibilicen en explotar en los alumnos su potencial cognitivo, sus actitudes, creencias ancestrales y costumbres para garantizar como seres humanos la agricultura en nuestro país.

\section{Referencias}

Avalos, R. (2014). Los Invernaderos como alternativa para optimizar el uso del suelo y elevar los rendimientos de cosechas. Trabajo de Grado para optar al título de Maestría en Formulación y Evaluación de Proyectos: Guatemala.

Barragán, H. (2010). Desarrollo, salud humana y amenazas ambientales: la crisis de la sustentabilidad. 1 a ed. -La Plata: Universidad Nacional de La Plata.

Bavaresco, A. (1994). Procesos metodológicos en la investigación. (2da. Ed). Academia Nacional de Ciencias Económicas y Servicios Bibliográficos de la Universidad del Zulia. Caracas. Venezuela.

Boff, L. (2013). La sostenibilidad. Editorial Sal Terrae. Impresión España.

Hurtado, J. (1998). Metodología de la investigación holística. Caracas, Fundación SYPAL.

Lama, R. (2007). Autogestión, manejarse a uno mismo para dirigir la 
Empresa. Perú: Universidad de San Martin de Porres.

Méndez, C. (2001). Metodología. Diseño y desarrollo del proceso de investigación. Tercera edición. McGraw-Hill. Colombia.

Marulanda, C. (2007). Manual técnico: La Huerta Hidropónica Popular. (3ra Ed). Santiago, Chile.

Marulanda, C. (2003). La Huerta Hidropónica Popular. Manual técnico. (3ra Ed). Santiago, Chile.

Rodríguez, D. (2004). Manual práctico de hidroponía. (4ta. Ed). Lima, Mekanobooks.

Rojas, B. (2010). Investigación cualitativa, fundamentos y praxis. Caracas: Fondo editorial de la Universidad Pedagógica Experimental Libertador.

Trujillo, E. (2000). Manejo de semillas, viveros y plantación Inicial. Litografía Ace Printer. Bogotá.

Universidad Pedagógica Experimental Libertador, Vicerrectorado de Investigación y Postgrado. (2012). Manual de grado de especialización y maestría y tesis doctorales. Caracas: Autor. 


\section{Luis Alfredo Díaz \\ e-mail: luis.a.godoy@hotmail.com}

Fecha de Nacimiento 20 de marzo de 1974, natural de

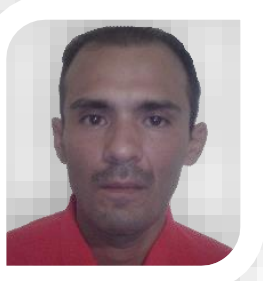

Barinas, Venezuela, 42 años de edad, soltero, dirección: Barinitas, municipio Bolívar, estado Barinas.

Estudios: Primaria: Escuela Básica Bolivariana

Barinitas. Secundaria: 7mo, Escuela Básica Bolivariana Barinitas. 8vo a 5 año. Pregrado: Lcdo. Educación: Dificultad de Aprendizaje en la Universidad Nacional Abierta, Barinas. Postgrado: Cursante Maestría: Educación Ambiente y Desarrollo, UPEL, Barinas. Desempeño: Docente de Aula integrada: Escuela Básica Bolivariana "Los Naranjos" Calderas, Barinitas, 2003-2008. Docente promotor de derecho Escuela Básica Bolivariana “Los Naranjos" NER. 187, 2008-2013. Docente defensor en la defensoría "Semillas del Futuro", 2013-2016.

El contenido de este manuscrito se difunde bajo una Licencia de Creative Commons ReconocimientoNoComercial-Compartirlgual 4.0 Internacional 